\title{
The Sport Concussion Assessment Tool: a systematic review
}

\author{
Aaron M. Yengo-Kahn, BS, ${ }^{1}$ Andrew T. Hale, BS, ${ }^{1}$ Brian H. Zalneraitis, BS, ${ }^{1}$ \\ Scott L. Zuckerman, MD, ${ }^{1,2}$ Allen K. Sills, MD, ${ }^{1,2}$ and Gary S. Solomon, PhD ${ }^{1,2}$ \\ ${ }^{1}$ Vanderbilt Sports Concussion Center, and ${ }^{2}$ Department of Neurological Surgery, Vanderbilt University School of Medicine, \\ Nashville, Tennessee
}

OBJECTIVE Over the last 2 decades, sport-related concussion (SRC) has garnered significant attention. Even with increased awareness and athlete education, sideline recognition and real-time diagnosis remain crucial. The need for an objective and standardized assessment of concussion led to the eventual development of the Sport Concussion Assessment Tool (SCAT) during the Second International Conference on Concussion in Sport in 2004, which is now in its third iteration (SCAT3). In an effort to update our understanding of the most well-known sideline concussion assessment, the authors conducted a systematic review of the SCAT and the evidence supporting its use to date.

METHODS English-language titles and abstracts published between 1995 and October 2015 were searched systematically across 4 electronic databases and a review was conducted in accordance with the Preferred Reporting Items for Systematic Reviews and Meta-Analyses (PRISMA) guidelines adapted for the review of a heterogeneous collection of study designs. Peer-reviewed journal articles were included if they reported quantitative data on any iteration of the SCAT, Standardized Assessment of Concussion (SAC), or modified Balance Error Scoring System (mBESS) data at baseline or following concussion in an exclusively athlete population with any portion older than 13 years of age. Studies that included nonathletes, only children less than 13 years old, exclusively BESS data, exclusively symptom scale data, or a non-SCAT-related assessment were excluded.

RESULTS The database search process yielded 549 abstracts, and 105 full-text articles were reviewed with 36 meeting criteria for inclusion. Nineteen studies were associated with the SAC, 1 was associated with the mBESS exclusively, and 16 studies were associated with a full iteration of the SCAT. The majority of these studies (56\%) were prospective cohort studies. Male football players were the most common athletes studied. An analysis of the studies focused on baseline differences associated with age, sex, concussion history, and the ability to detect an SRC.

CONCLUSIONS Looking toward the upcoming Concussion in Sport Group meeting in fall 2016, one may expect further revision to the SCAT3. However, based on this systematic review, the authors propose further, in-depth study of an already comprehensive concussion test, with acute, diagnostic, as well as long-term use.

http://thejns.org/doi/abs/10.3171/2016.1.FOCUS15611

KEY WORDS sideline assessment; Sport Concussion Assessment Tool; Standardized Assessment of Concussion; modified Balance Error Scoring System; sport-related concussion

$\mathrm{O}$ ver the last 2 decades, sport-related concussion (SRC) has emerged as a major public health problem with many stakeholders. ${ }^{4,42,56}$ The Centers for Disease Control and Prevention estimate up to 3.8 million instances of SRC in any given year. ${ }^{17,27}$ Even with increased awareness and attempts at athlete education,, 726 sideline recognition and real-time diagnosis by team physician or training staff remains crucial. ${ }^{17,36,39}$
Recognition and diagnosis of SRC based on symptoms alone may be challenging given the variability in symptom reporting. ${ }^{40}$ Due to the subjective nature of symptom endorsement, athletes may fail to disclose or purposefully hide symptoms from sideline staff to remain in the game. ${ }^{20,25}$ The need for an objective and standardized assessment of concussion, including brief screening of neurocognitive and neurological functions in addition to

ABBREVIATIONS AUC = area under the curve; GCS = Glasgow Coma Scale; $\mathrm{mBESS}=$ modified Balance Error Scoring System; $\mathrm{mTBI}=$ mild TBI; NFL = National Football League; $\mathrm{NHL}=$ National Hockey League; PCSS = Post-Concussion Symptom Scale; PRISMA = Preferred Reporting Items for Systematic Reviews and Meta-Analyses; ROC = receiver operating characteristic; SAC = Standardized Assessment of Concussion; SCAT = Sport Concussion Assessment Tool; SRC = sport-related concussion; TBI $=$ traumatic brain injury.

SUBMITTED December 1, 2015. ACCEPTED January 15, 2016. INCLUDE WHEN CITING DOI: 10.3171/2016.1.FOCUS15611. 
symptom assessment, led to the development of the Standardized Assessment of Concussion (SAC) in 1997. ${ }^{31-33}$ During the Second International Conference on Concussion in Sport in 2004, the SAC was combined with a standardized symptom scale (the Post-Concussion Symptom Scale [PCSS]), sport-specific orientation questions (i.e., modified Maddocks' questions), on-field markers of concussion (e.g., amnesia, loss of consciousness), and guidelines for a systematic, stepwise return to play to create the first iteration of the Sport Concussion Assessment Tool (SCAT). ${ }^{34}$ While the primary purpose of the SCAT was to standardize sideline diagnosis of concussion, it also served as a tool for patient education. ${ }^{34}$

At the third meeting of the Concussion in Sport Group, ${ }^{35}$ the SCAT underwent revisions to include the additions of: 1) the Glasgow Coma Scale (GCS); 2) alternate word lists for the immediate and delayed recall portion of the SAC; and 3) a modified version of the Balance Error Scoring System (mBESS, consisting of 3 stances performed on a hard surface). ${ }^{35}$ Although the SCAT2 was more labor intensive and required greater time to administer, it was designed for both sideline and serial use to track an athlete's recovery during subsequent clinical assessments..$^{21,35}$ In the most recent revision (SCAT3), published in 2013, ${ }^{14}$ additional physical/objective signs (i.e., blank/vacant look, cervical stability assessment) were added with the option of using either foam stances or a timed tandem gait task for postural stability assessment. The SAC and PCSS remained unchanged in the SCAT3 from its original iteration. ${ }^{14}$ In addition, a SCAT3 version tailored to children (age $<13$ years) was introduced, the Child-SCAT3. ${ }^{36}$

Prior to the release of the SCAT3, the SCAT2 became widely used by certified athletic trainers and team physicians. ${ }^{21,39}$ Administration of the SCAT2 had been made more efficient with the advent of smartphone and tablet versions. ${ }^{5}$ Because few generalizable studies have been conducted to empirically support the use of the SCAT2, questions were raised regarding its sensitivity and specificity, which may have led to the creation of the SCAT3.$^{15,40}$

Although Guskiewicz et al. presented a concise review of the evidence base upon which the SCAT3 was created, ${ }^{14}$ it has been 3 years since this review and 8 years since the release of the SCAT2. Further, both the National Football League (NFL) and the National Hockey League (NHL) have used versions of the SCAT league-wide for sideline assessment of suspected concussion. ${ }^{39}$ Given the increased use of the SCAT, we performed a systematic review of the current literature related to the SCAT and its objective components. The a priori objectives of the systematic review were to: 1) concisely summarize all published data on the 3 iterations of the SCAT, as well as its major components (the SAC and mBESS); 2) present aggregate normative data for the major components of the SCAT broken down by age and sex; and 3) identify areas for improvement for the next iteration of the SCAT.

\section{Methods \\ Literature Search}

Titles and abstracts in the English language published between 1995 and October 2015 were searched systemati- cally across the electronic databases PubMed, PsychInfo, The Cumulative Index to Nursing and Allied Health Literature (CINAHL), and Embase. To complete the search, the reference lists from review articles were assessed for pertinent studies that may have been overlooked. Key words and search terms included brain concussion, mild traumatic brain injury, Sideline Concussion Assessment Tool, standardized assessment of concussion, concussion resolution index, balance error scoring system, and neurocognitive or neuropsychological testing. In addition, variations and acronyms of these terms (i.e., BESS, SCAT, SAC, mTBI) were included in search syntax. An example of the search syntax is available in Fig. 1. The Preferred Reporting Items for Systematic Reviews and Meta-Analyses (PRISMA) ${ }^{38}$ were adapted to review a heterogeneous collection of literature.

\section{Inclusion and Exclusion Criteria}

A priori inclusion criteria were: 1) peer-reviewed journal articles published after 1995; 2) individuals greater than 13 years of age; 3 ) outcomes (primary or otherwise) reported included a quantitative analysis of the SCAT, SCAT2, SCAT3, or a major objective component of these assessments (defined as the SAC or mBESS); and 4) the data reported were obtained at baseline or postconcussion.

However, part of our a priori inclusion criteria was modified. For the purpose of this review, we allowed for the inclusion of athletes younger than age 13 years (e.g., studies with an age range of 5-23 years). These studies were considered important as a large number of athletes included were older than 13 years of age. We did not include studies in which the athletes were all younger than 13 as the Child-SCAT3 has since been created for this age group.

Six exclusionary criteria were applied: 1) the investigation did not perform any testing with a version of the SCAT, SAC, or mBESS (e.g., only computerized or traditional paper and pencil neuropsychological testing, EYETRAC Advance, King-Devick, or simple reaction time task); 2) only the BESS was assessed and reported alone, because the mBESS is used in the SCAT (3-stance firm surface only), rather than the full BESS, which utilizes foam and firm surface stances; 3) solitary assessment of the PCSS or other similar symptom scales were excluded due to heterogeneous content and rating formats, and concussion symptom scales were reviewed thoroughly elsewhere; ${ }^{1}$ ) nonathlete (e.g., motor vehicle accident-related concussion, healthy adults) and mixed (athlete plus nonathlete) populations were excluded to ensure a focus on sport-related concussion; 5) the type of publication was a conference abstract (without full data set), review article, editorial, or letter to the editor; and finally 6) we excluded the GCS component of the SCAT due to its overwhelming evidence in the traumatic brain injury (TBI) population and paucity in athletic populations.

\section{Data Extraction}

Following compilation of online search results, record titles and abstracts were screened by one of the authors (A.T.H.) with oversight by the first author (A.M.Y.K.). 


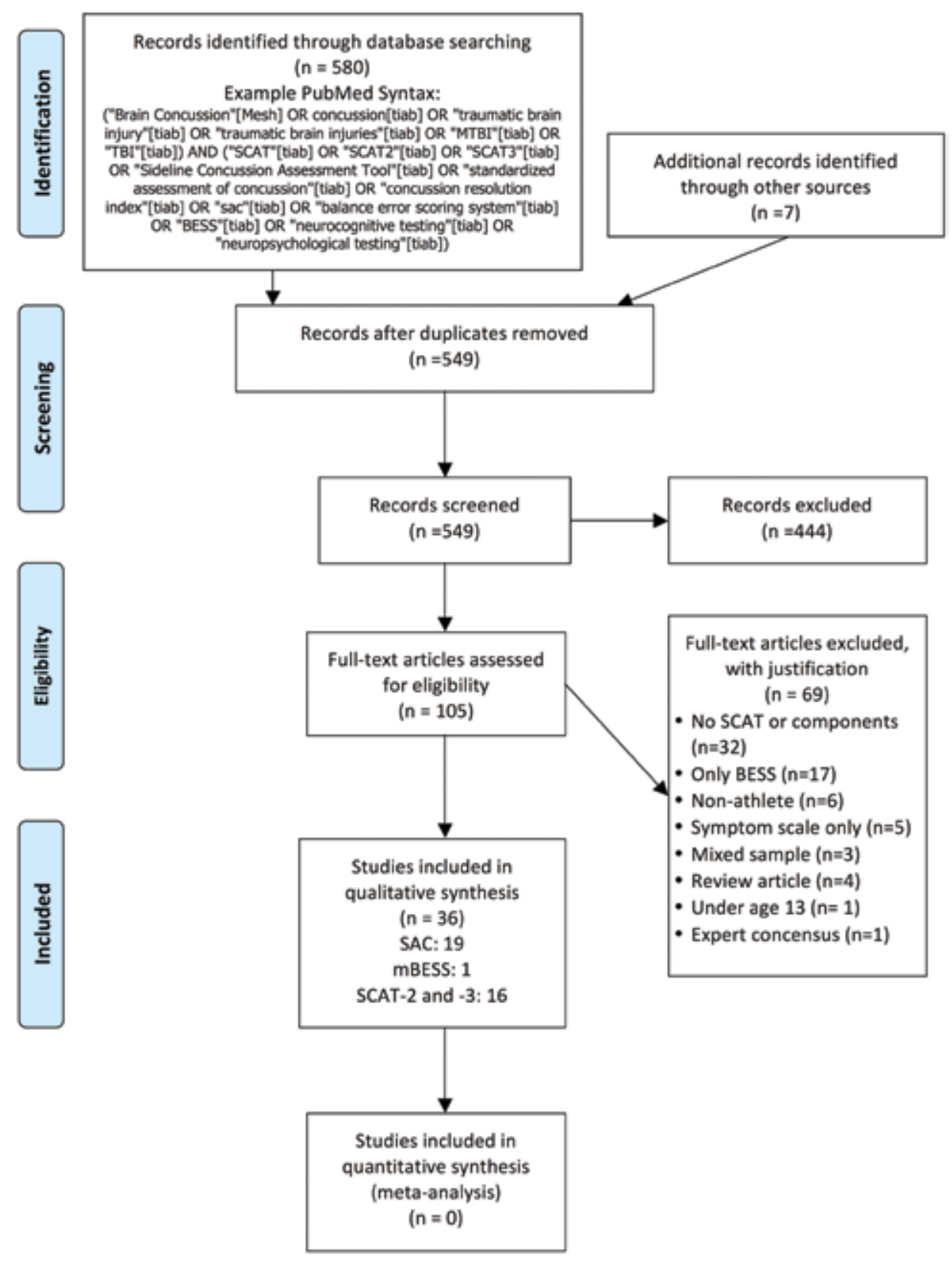

FIG. 1. Flow chart of the systematic review process.

Those articles determined to have potential to meet inclusion and exclusion criteria were passed through to full-text review by 4 authors (A.T.H., B.H.Z., S.L.Z., and A.M.Y.K.). The first author (A.M.Y.K.) reviewed all included and excluded studies for an added level of verification. Disagreements on the inclusion of a study were settled by joint discussion among senior authors (A.M.Y.K., S.L.Z., A.K.S., and G.S.S.). Fig. 1 illustrates the literature review process. Following full-text review, the authors extracted data into evidence tables, which were used for the synthesis within the results section.

\section{Results}

Of the 36 included articles, the majority were cohort studies $(\mathrm{n}=22)$, of which 20 were prospective and 2 were retrospective. Twelve cross-sectional studies and 2 case series were reviewed. Eighteen articles examined athletes exclusively at baseline, 1 examined athletes following concussion, and 17 reported both baseline and postconcussion data. All studies meeting criteria for review are listed in Table 1.

\section{Standardized Assessment of Concussion General Results}

Nineteen studies were found that met inclusion criteria and were associated with the SAC alone or discussed the SAC and other tests unrelated to the SCAT. Participant athletes were assessed primarily at baseline $(n=9086)$ with fewer having been assessed after concussion $(\mathrm{n}=$ 
TABLE 1. All studies meeting criteria for inclusion

\begin{tabular}{|c|c|c|c|}
\hline Authors \& Year & Study Design & $\begin{array}{l}\text { Level of } \\
\text { Evidence* }\end{array}$ & Test \\
\hline Galetta et al., 2015 & Prospective & $1 b$ & SAC \\
\hline Wang et al., 2015 & Prospective & $1 b$ & SAC \\
\hline Galetta et al., 2013 & Cross-sectional & 4 & SAC \\
\hline McCrea et al., 2013 & Prospective & $1 b$ & SAC \\
\hline Zimmer et al., 2013 & Cross-sectional & 4 & SAC \\
\hline Gysland et al., 2012 & Prospective & $1 b$ & SAC \\
\hline Sabin et al., 2011 & Retrospective & $2 b$ & SAC \\
\hline Hunt \& Ferrara, 2009 & Cross-sectional & 4 & SAC \\
\hline Miller et al., 2007 & Case series & 4 & SAC \\
\hline Valovich McLeod et al., 2006 & Prospective & $1 b$ & SAC \\
\hline Valovich McLeod et al., 2004 & Prospective & $1 b$ & SAC \\
\hline McCrea et al., 2003 & Prospective & $1 b$ & SAC \\
\hline Valovich et al., 2003 & Prospective & $1 b$ & SAC \\
\hline Daniel et al., 2002 & Prospective & $1 b$ & SAC \\
\hline McCrea et al., 2002 & Prospective & $1 b$ & SAC \\
\hline Barr \& McCrea, 2001 & Prospective & $1 b$ & SAC \\
\hline McCrea, 2001 & Prospective & $1 b$ & SAC \\
\hline McCrea et al., 1998 & Case series & 4 & SAC \\
\hline McCrea et al., 1997 & Prospective & $1 b$ & SAC \\
\hline King et al., $2014^{24}$ & Cross-sectional & 4 & mBESS \\
\hline Schneider et al., 2010 & Cross-sectional & 4 & SCAT \\
\hline Shehata et al., 2009 & Cross-sectional & 4 & SCAT \\
\hline Baker et al., 2015 & Retrospective & $2 b$ & SCAT2 \\
\hline Glaviano et al., 2015 & Cross-sectional & 4 & SCAT2 \\
\hline Putukian et al., 2015 & Prospective & $1 b$ & SCAT2 \\
\hline Snyder \& Bauer, 2014 & Cross-sectional & 4 & SCAT2 \\
\hline Zimmer et al., 2015 & Cross-sectional & 4 & SCAT2 \\
\hline King et al., 2013 & Prospective & $1 b$ & SCAT2 \\
\hline Weber et al., 2013 & Prospective & $1 b$ & SCAT2 \\
\hline Echlin et al., 2012 & Prospective & $1 b$ & SCAT2 \\
\hline Jinguji et al., 2012 & Cross-sectional & 4 & SCAT2 \\
\hline Valovich McLeod et al., 2012 & Cross-sectional & 4 & SCAT2 \\
\hline Echlin et al., $2010^{10}$ & Prospective & $1 b$ & SCAT2 \\
\hline Hanninen et al., 2015 & Cross-sectional & 4 & SCAT3 \\
\hline King et al., 2015 & Prospective & $1 b$ & SCAT3 \\
\hline Vernau et al., 2015 & Prospective & $1 b$ & SCAT3 \\
\hline
\end{tabular}

* Using the Oxford Center for Evidence-Based Medicine Levels of Evidence (http://www.cebm.net/ocebm-levels-of-evidence/).

943). The vast majority of athletes studied were male (Fig. 2B). Of the 19 athlete studies, 12 were conducted with football players, and the remaining populations described were composed of athletes from multiple sports including hockey, rugby, soccer, and volleyball (Fig. 2A). Summary information for studies utilizing the SAC can be found in Table 2.

\section{Effect of Age}

The effects of age on the SAC have been reported in the literature..$^{11,18,33}$ In one of the initial studies of the SAC,
McCrea et al. ${ }^{33}$ found no age effect on SAC scores between male high school and college football players. Similarly, Hunt and Ferrara ${ }^{18}$ found that SAC scores in 198 male high school football players did not significantly increase from 9th to 12th grade. Most recently, however, Galetta et al. found that increasing age was associated with significantly increased SAC scores $(\mathrm{p}<0.001$, linear regression model) for a group of 332 athletes from various sports. Notably, the cohort ranged in age from 5 to 23 years. ${ }^{11}$

\section{Effect of Sex}

Various authors have commented on the potential as-

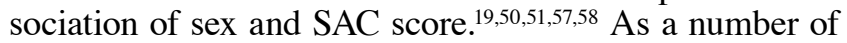
the early studies utilizing the SAC in an athlete population were conducted on exclusively male football teams, ${ }^{29,31-33}$ sex differences were first reported in 2006 during a psychometric evaluation of the SAC. Valovich McLeod et al. demonstrated that in a multisport athletic population aged 9-14, males performed significantly worse on the SAC than females (26.22 vs 27.50; $p=0.035) .{ }^{51}$ Similar findings were reported in a 2012 publication of SCAT2 normative data by Valovich McLeod et al. ${ }^{50}$ who found males performed worse than females (26.4 vs 26.9; $\mathrm{p}=0.004)$ on the SAC in a high school-aged, multisport cohort (mean age approximately 15 years). In a similar study, Jinguji et al. ${ }^{19}$ found comparable sex associations and further attributed them to particular subtests of the SAC. Specifically, females in all age groups (13-19 years old) performed significantly better on SAC components assessing concentration $(\mathrm{p}=0.01)$. Additionally, females outperformed males within the same age group (13-15 or 16-19 years old) on the immediate memory component $(\mathrm{p}=0.02-0.04)$.

Despite these early reports, more recent studies with athletes have been inconsistent in demonstrating a relationship between sex and SAC scores. Zimmer et al., ${ }^{58}$ in a study of SAC scores across various collegiate sport teams, reported no sex differences on the SAC. Yet, in a second study of college athletes by Zimmer et al., ${ }^{57}$ females performed significantly better (27.63 vs $26.97 ; p=0.004)$ than males.

\section{Detecting Concussion}

The use of the SAC to detect SRC began in the 1990s with a series of studies of high school and college football players. From 1997 to 2003, there were 8 peer-reviewed articles examining SAC performance in football players after a concussion with comparisons to individual baseline or control players. ${ }^{3,6,28,29,31-33}$ All of these studies demonstrated a significantly decreased total SAC score when compared with individual baseline or uninjured controls. The largest sample of concussed collegiate athletes $(\mathrm{n}=$ 94) was presented by McCrea et al. ${ }^{29}$ in 2003 . Immediately after injury, all football players scored an average of 2.94 points below baseline (95\% CI -4.39 to -1.50$)$. In most of these similar studies, players were reevaluated with the SAC at 48 hours postinjury and were found to have SAC scores that were not significantly different from baseline. ${ }^{28,29,33}$

The sensitivity and specificity of the SAC has been reported sparsely. Barr and McCrea reported that a 1-point drop on the SAC was $94 \%$ sensitive and $76 \%$ specific for 


\section{A SAC Studies by Sport}

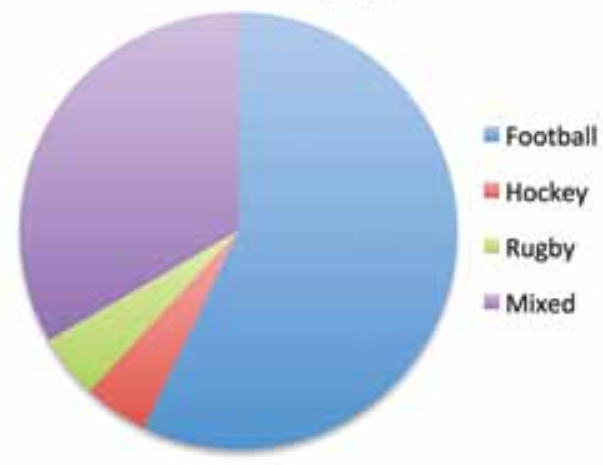

C SCAT Studies by Sport

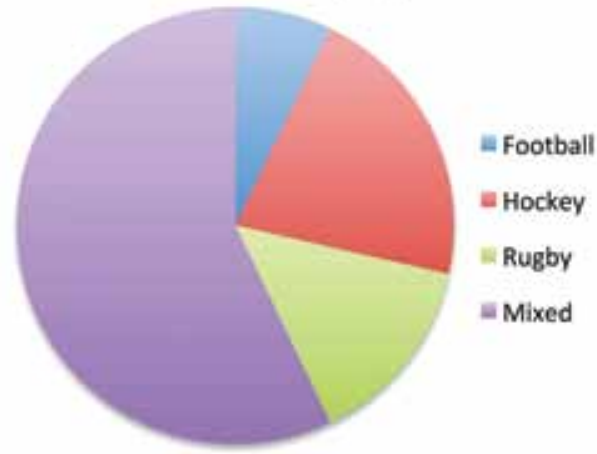

\section{B Gender of SAC Study Population}

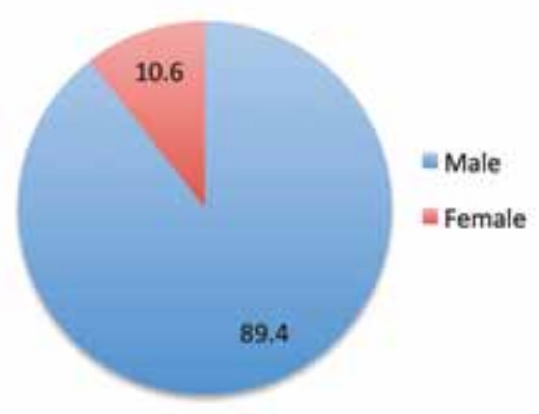

D Gender of SCAT Study Population

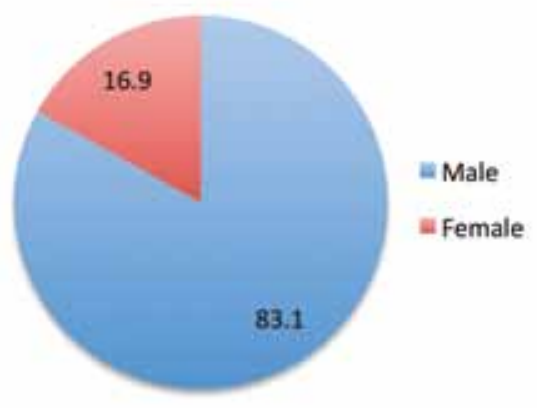

FIG. 2. Graphic representation of themes in study population demographics for the SAC (A and B) and SCAT (C and D).

concussion in a mixed sample of high school and collegiate football players. ${ }^{3}$ The authors also presented receiver operating characteristic (ROC) curves and found the area under the curve (AUC) to range from $0.911 \pm 0.028$ (SEM) to $0.939 \pm 0.021(\mathrm{SEM})$ depending on whether a regression analysis or a $90 \%$ reliable change index, respectively, was used. ${ }^{3}$ Similarly, McCrea reported a single point drop on the SAC to be $95 \%$ sensitive and $76 \%$ specific for diagnosing sideline SRC immediately after a head impact in a mixed high school and collegiate sample. ${ }^{28}$ However, in a more recent study of an age-diverse population (age 5-23), the ROC curve constructed based on baseline and postinjury SAC scores of 12 concussed athletes was found to have an AUC of 0.68 , utilizing a logistic regression model accounting for age, markedly lower than the previous study. ${ }^{11}$

Only more recently has the SAC been evaluated outside of the realm of football, in other sport and nonathlete populations. In a recent study of 32 rugby players ( 27 male), Putukian et al. found no difference between postinjury and baseline SAC scores when tested $0.52 \pm 1.18$ (SD) days after concussion $(p>0.1){ }^{41}$

Recently, authors have used the SAC in conjunction with more complex methods of concussion detection. Wang and coauthors ${ }^{54}$ compared 18 concussed football players to 19 controls and tested them with the SAC and MRI to detect cerebral blood flow at baseline and multiple time points following SRC. Their data demonstrated that decreased cerebral blood flow may persist following symptom resolution and normalization of the SAC score.

\section{Modified Balance Error Scoring System General Results}

Only 1 study was found that met all inclusion criteria and was associated solely with the mBESS in a sports population (or discussed the mBESS and other tests that do not comprise the SCAT). Typically, however, the mBESS is discussed within the context of the SCAT2 or SCAT3. The following results contain data from studies reported in Tables 2 and 3.

\section{Effect of Age}

Age appeared to have a variable association with mBESS scores. In Snyder and Bauer's cohort of 761 student athletes aged 9-18, athletes in the younger age group (age 9-10) incurred more errors on the mBESS than those athletes in either the $15-16$ or $17-18$ age group. ${ }^{47}$ Furthermore, in a separate study of high school athletes, mBESS scores improved by grade from 9th to 12 th, with 10th, 11 th, and 12th graders performing significantly better than 9th graders $(\mathrm{p}=0.002){ }^{50}$

\section{Effect of Sex}

Sex differences on the mBESS in athletes at baseline have also been described. Snyder and Bauer also found no sex difference on the mBESS in their youth and high school athlete sample. ${ }^{47}$ In contrast, 3 studies suggested that females perform better than males on the mBESS. Valovich McLead and coauthors found that high school female athletes outscored high school male athletes (27.3 vs 26.6; 


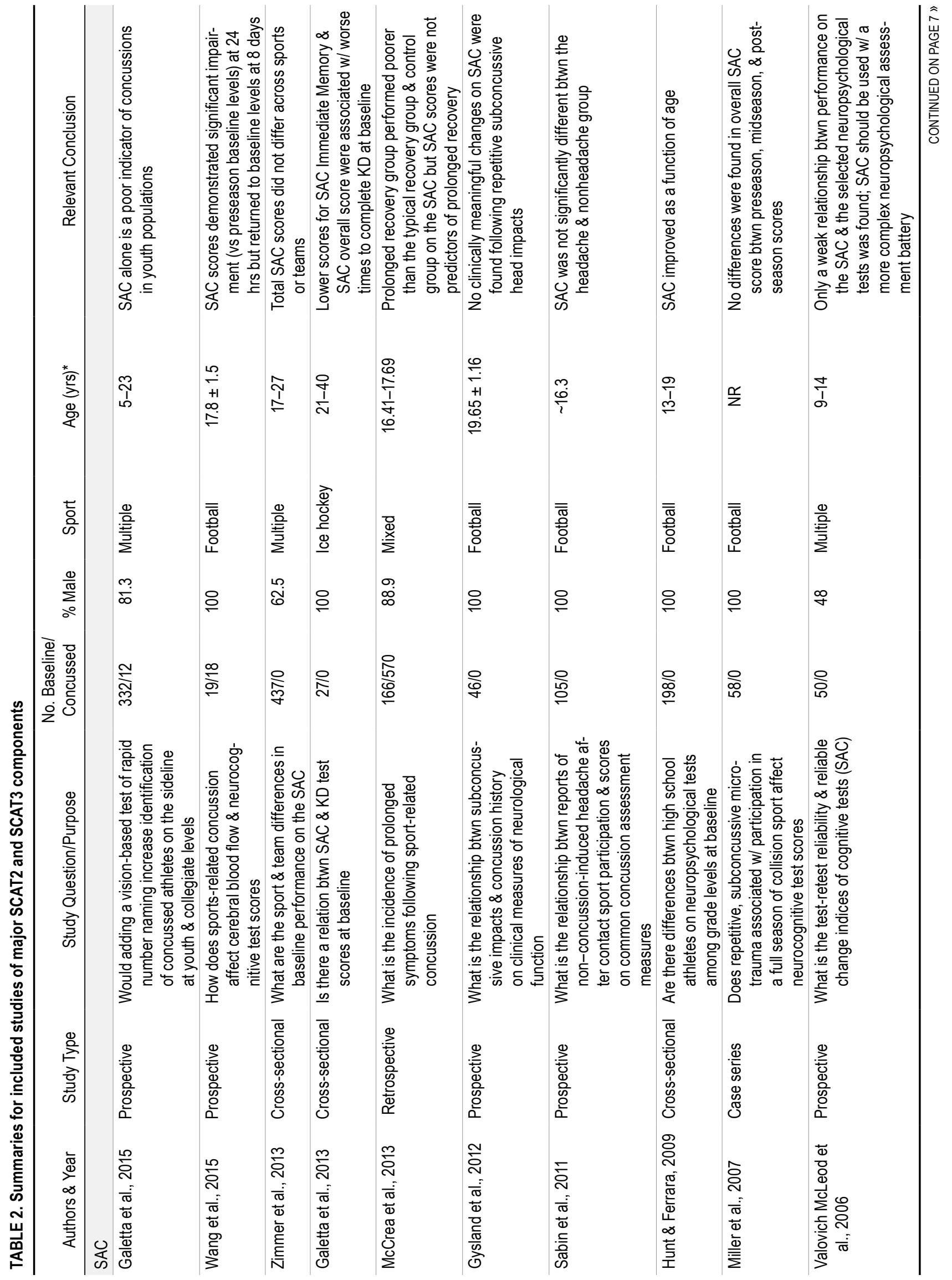




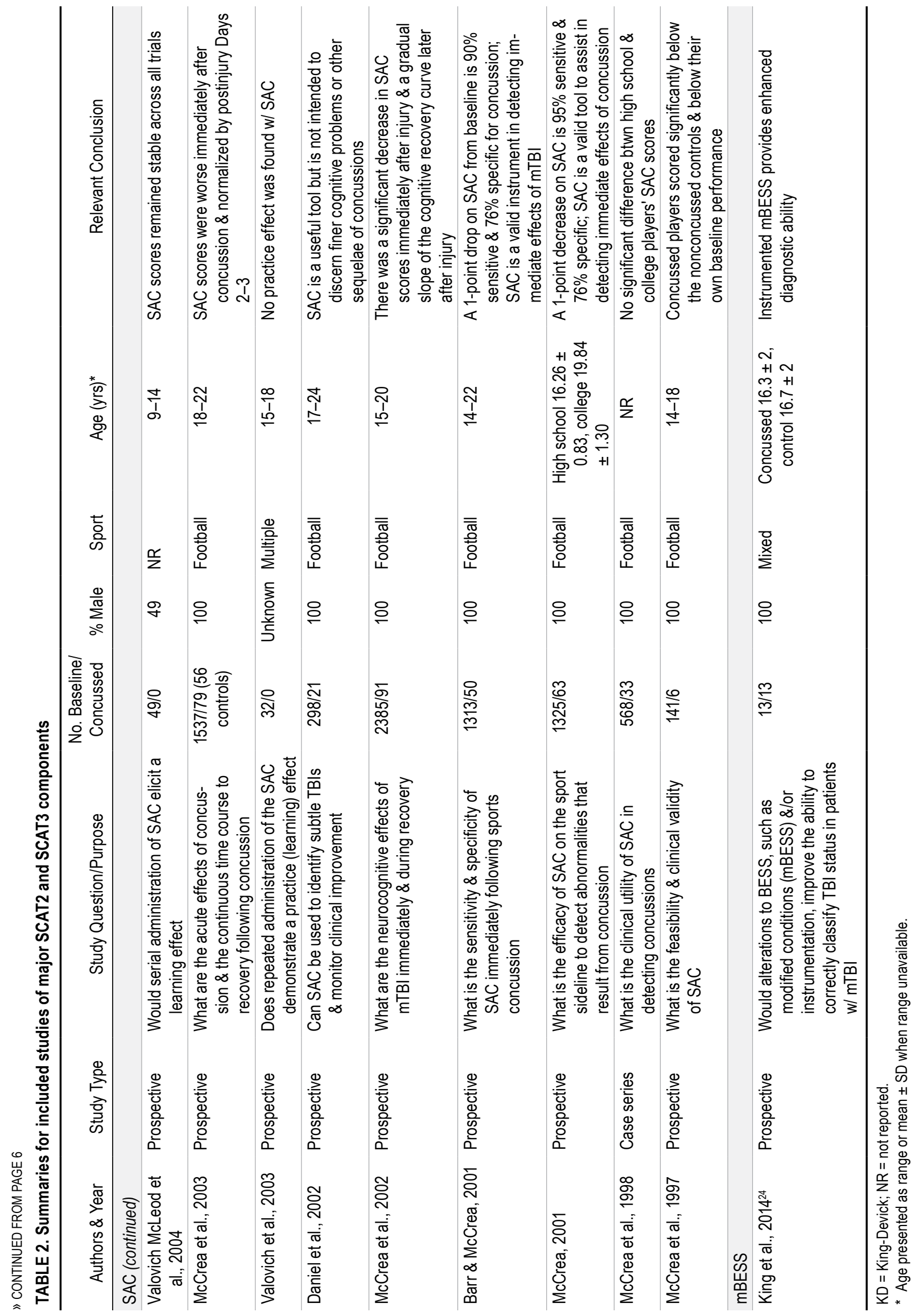




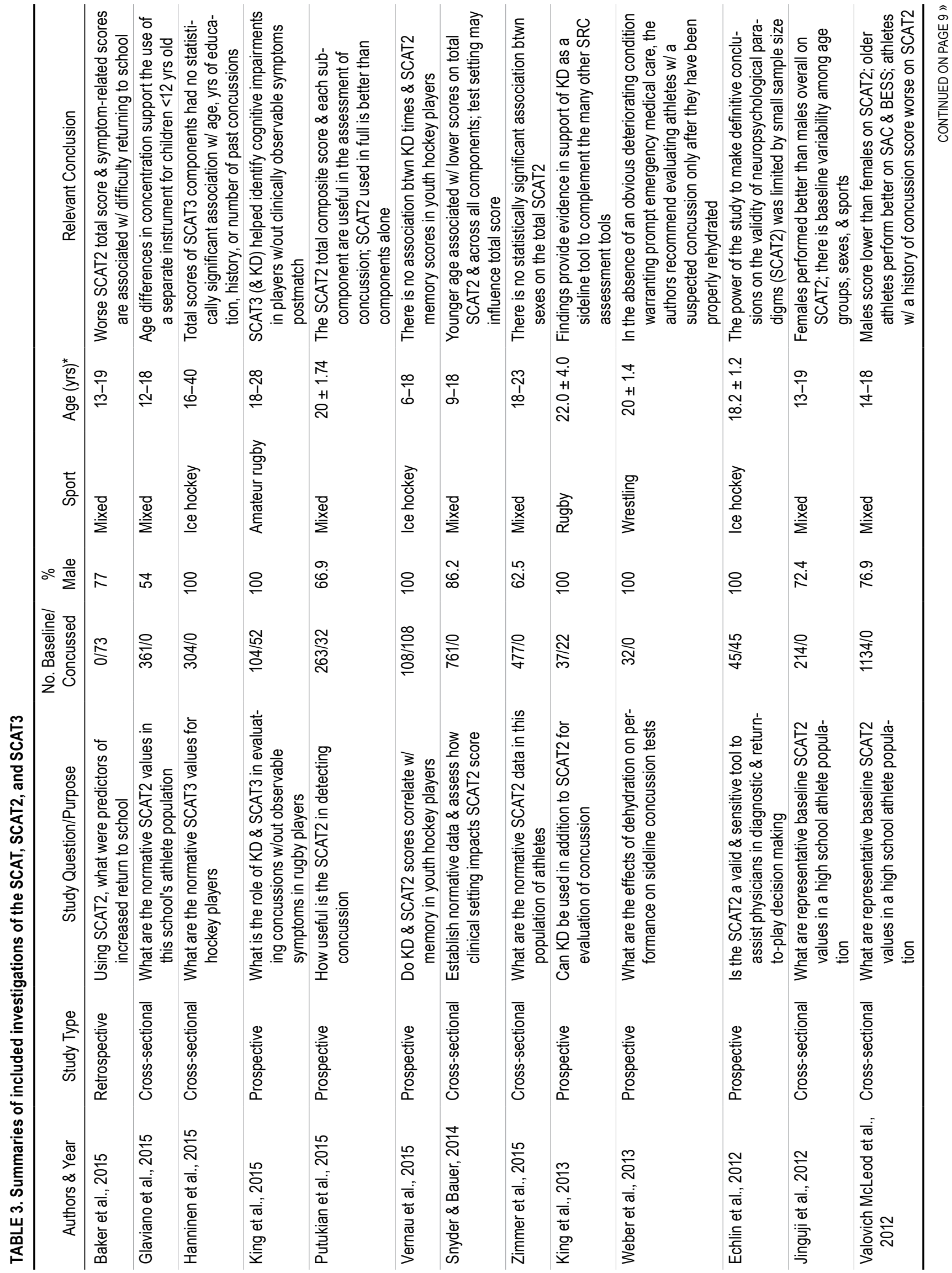




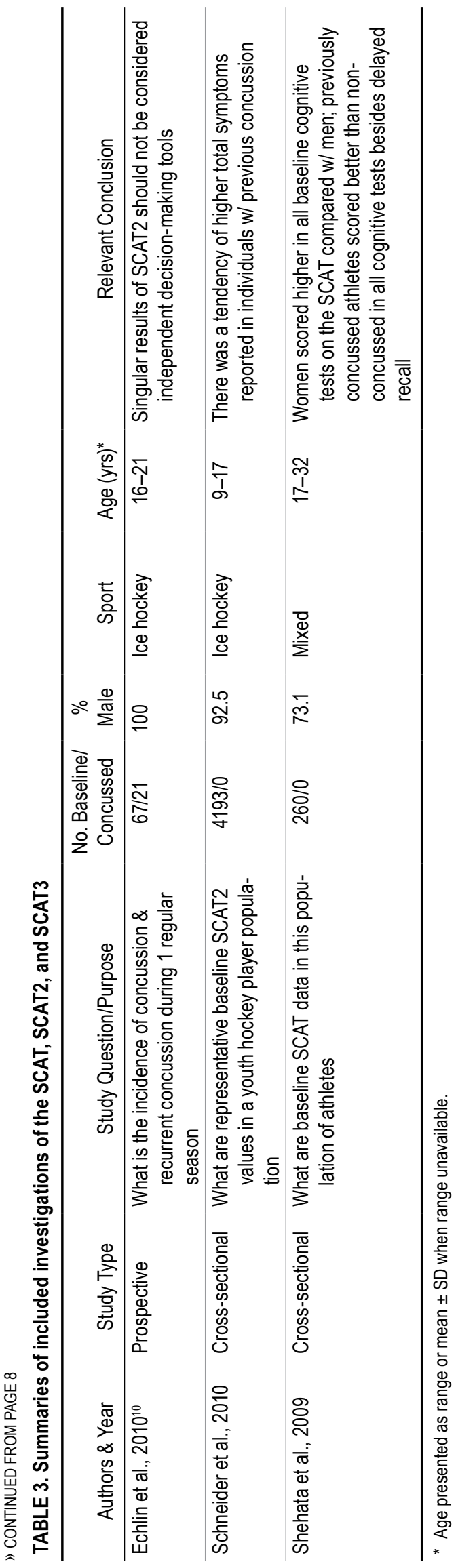

$\mathrm{p}<0.001)$ on the mBESS. ${ }^{50}$ Jinguji et al. reported a similar relationship of female performance superiority (26.85 vs 25.43; $\mathrm{p}=0.01$ ) in a separate cohort of high school athletes. ${ }^{19}$ Glaviano et al. also found females recorded fewer errors than males (total score 27.2 vs $26.6 ; p=0.0043$ ) on the mBESS in a group of athletes aged $12-18 .^{13}$

\section{Detecting Concussion}

In a group of 32 collegiate athletes, Putukian et al. provided the first evidence that mBESS scores decline from baseline following concussion (26.13 vs 24.54 ; $p<0.05) .{ }^{41}$ In an effort to improve concussion detection with the mBESS, King et al. ${ }^{24}$ added instrumentation in the form of an inertial sensor (accelerometer and gyroscope). The instrumented mBESS was found to be significantly better at detecting concussion (AUC $=0.81,95 \%$ CI 0.64-0.990) than the noninstrumented mBESS (AUC $=0.64,95 \% \mathrm{CI}$ $0.42-0.86)$.

\section{Sport Concussion Assessment Tool General Results}

Sixteen studies met inclusion/exclusion criteria and were associated with the first, second, or third edition of the SCAT alone or discussed the SCAT ( 2 or 3 ) and other tests unrelated to the SCAT. Of these 16 studies, 11 assessed the SCAT2, 3 used the SCAT3, and only 2 assessed the original SCAT. Of the excluded studies, 2 studies had a mixed population of both athletes and nonathletes and 1 study used entirely nonathletes. As with the SAC, the majority of participants were assessed at baseline rather than postconcussion (8360 vs 353, respectively). A large fraction of athletes were studied at baseline in an attempt to establish normative data. Two studies have been conducted to establish SCAT ${ }^{45,46}$ normative data, 5 for the SCAT2, ${ }^{13,19,47,50,58}$ but only 1 for the SCAT $3 .{ }^{16}$ These studies have reported on variations of the SCAT across ages, sex, and with concussion history. The majority of these studies used mixed sports populations (Fig. 2C), and the athletes studied were $>85 \%$ male (Fig. 2D).

\section{Effect of Age}

Despite a wide age range (17-32 years of age), the first normative study of the original SCAT, by Shehata et al., did not include an analysis of variation of the SCAT by age. ${ }^{46}$ In the largest study to provide normative SCAT data, Schneider et al..$^{45}$ administered a baseline SCAT to over 4000 youth hockey players. Although the authors did not report the effect of age on SCAT scores quantitatively, they did report that a greater percentage of older female hockey players (age 15-17) reported higher total symptoms at baseline compared with the younger female players (age 9-10).

The association between the SCAT2 and age was first described by both Jinguji et al. ${ }^{19}$ and Valovich McLeod et al. ${ }^{50}$ in their publications of normative SCAT2 data. Jinguji et al. divided their group of high school athletes into 2 age cohorts (13-15 and 16-19). The authors found a trend toward the younger age group reporting fewer symptoms (19.14 vs $20.32 ; \mathrm{p}=0.06)$ and performing better than the older group on the delayed recall portion of the SCAT2. 
Additionally, the younger group recorded a higher total SCAT2 score (89.37 vs 88.66; $\mathrm{p}$ value not reported), but none of these findings reached statistical significance. ${ }^{19}$ Valovich McLeod et al. reported normative data from a large cohort of 1134 high school athletes from various sports. The total SCAT2 score was found to increase each grade from 9th to 12th with both the 11th and 12th graders scoring significantly better than 9 th graders $(88.7$ vs 89.0 vs 86.9 , respectively; $p=0.001$ ). Secondary analysis of component scores found this difference was likely related to significantly better SAC ( $p<0.001)$ and mBESS ( $p$ $=0.002$ ) scores for 10th, 11th, and 12th graders compared with 9th graders. ${ }^{50}$

Following these initial normative data publications, Snyder and Bauer published SCAT2 normative data of 761 athletes with a wider age range of 9-18 years. A significant positive, linear association between age and total SCAT2 scores was found $(\mathrm{p}<0.001){ }^{47}$ These differences were attributed specifically to the balance and cognitive components in which younger athletes (ages 9-10) performed significantly worse than older athletes (aged 17-18). However, when the authors controlled for test setting and sex, only total SCAT2 score and the balance component were significantly associated with age, with younger athletes performing worse. ${ }^{47}$ In contrast, Glaviano et al. gave the baseline SCAT2 to 361 middle and high school athletes and found that although the total SCAT2 score did not vary with age $(p=0.21)$, specific SCAT2 component scores appeared age dependent. ${ }^{13}$ Specifically, the authors found that 12-year-olds performed worse on the concentration component compared with 15 - and 18 -year-olds (3.3 vs 3.9 vs 4.2 , respectively; $p=0.01$, yet no other statistically significant differences related to age were found. ${ }^{13}$

While the studies mentioned above are almost exclusively in high school athletes, Zimmer et al. ${ }^{57}$ reported SCAT2 normative data for collegiate athletes. In a population of 477 college athletes from various sports aged $18-23$, an average total SCAT2 score of $91.08 \pm 5.60$ is reported. Although the authors correctly commented that this score alone was greater than that reported in studies of high school students, Zimmer et al. did not provide analysis of their sample by age. ${ }^{57}$ To date, Hanninen et al. is the only group to publish normative data for the SCAT3. In their population of 304, mostly Finnish, professional hockey players aged 16 to 40 years old, there was no association of any portion of the SCAT3 with age. ${ }^{16}$

\section{Effect of Sex}

The potential for sex effects on each iteration of the SCAT has been well described. For the original SCAT, both Shehata et al. ${ }^{46}$ and Schneider et al ${ }^{45}$ found extensive differences between males and females. In a group of 260 collegiate ice hockey, football, and wrestling athletes (age 17-32), Shehata et al ${ }^{46}$ reported a higher symptom score at baseline ( 6.39 vs 3.52 ; p value not reported), but a better performance on all baseline cognitive tests for females compared with males. However, no statistical comparative analysis was performed to determine significance. ${ }^{46}$ Similarly, Schneider et al. found that sex differences on symptom reporting varied with age group, in which about the same percentage of older females (64.3\%, age 13-14) reported symptoms as the oldest males (64.5\%, age 13-14), but younger males (age 11-12) reported more symptoms than same-aged females. Additionally, the authors found that a relatively young group of females (age 11-12) were able to all complete the months-in-reverse task, while only $50 \%$ of males up to age 14 could complete it, a result the authors suggested as a sex difference. Again, it is important to point out that no quantitative analysis was conducted to assess for statistical significance. ${ }^{45}$

Sex differences were also a centerpiece of both Valovich McLeod et al.'s and Jinguji et al.'s SCAT2 normative data publications in 2012. Jinguji et al. found that male high school athletes reported slightly fewer symptoms (19.79 vs $19.64 ; p=0.38$, higher score represented fewer symptoms), but performed significantly worse than females on the SCAT2 overall (88.39 vs $90.81 ; \mathrm{p}<0.01) .{ }^{19}$ This result was reinforced by Valovich McLeod et al. in a large sample of 1134 athletes, with results indicating that high school males performed significantly worse than females on the SCAT2 overall ( 87.7 vs $88.7 ; p=0.03) .{ }^{50}$

Conversely, 3 subsequent studies reported no statistically significant difference in total SCAT2 scores between males and females. Snyder et al. reported no sex difference on the total SCAT2 score (or SAC or mBESS), but did find that females reported significantly more symptoms (and with great severity) at baseline than males across a wide age range (9-18 years old). ${ }^{47}$ Similarly, Glaviano et al. found no sex difference in high school athletes on the total SCAT2 score $(p=0.463)$, but females reported more symptoms $(p=0.007) .{ }^{13}$ Finally, Zimmer et al. found no significant main effect of sex on total SCAT2 score or symptoms in a cohort of college athletes. ${ }^{57}$

\section{Concussion History}

There have been numerous publications that have examined normative SCAT data stratified by concussion history; results have varied widely. When Shehata et al. ${ }^{46}$ investigated the association of previous concussion history and the original SCAT, previously concussed individuals appeared to perform better on all cognitive testing with the exception of the delayed recall component. For example, $61 \%$ of previously concussed athletes were able to score 6 digits on the digits-reverse test while only $46 \%$ of never concussed athletes were able to equal this level of performance. Similarly, Schneider et al. ${ }^{45}$ reported that among 11-12 year olds, $100 \%$ of previously concussed athletes were able to complete the months-in-reverse task while only $40.5 \%$ of never concussed athletes achieved this. The authors also reported a tendency for previously concussed athletes to report more symptoms with headaches being the most common.

In high school athletes, Valovich McLeod et al. ${ }^{50}$ found that previously concussed athletes performed worse on the SCAT2 overall ( 87.0 vs $88.7 ; \mathrm{p}<0.001)$. This difference may be attributable to previously concussed athletes reporting a large total symptom burden $(p<0.001)$. These differences noted at the high school level do not appear to transfer to the college or professional level as both Zimmer et al. ${ }^{57}$ and Hanninen et al. ${ }^{16}$ reported no effect of concussion history on SCAT2 performance. 


\section{Detecting Concussion}

In contrast to the SAC, few studies have assessed the full SCAT and its iterations to determine sensitivity or specificity for detecting concussion on the sideline. $\mathrm{Pu}$ tukian et al ${ }^{41}$ calculated the sensitivity and specificity of the SCAT2 utilizing a prospective cohort study design with 263 collegiate athletes, 32 of whom suffered concussions. A 3.5-point drop from baseline was found to be $96 \%$ sensitive and $81 \%$ specific (AUC $=0.93$ ) to detect concussion, while using a cutoff score of 74.5 proved to be $83 \%$ sensitive and $93 \%$ specific (AUC $=0.91$ ). Conversely, in a small study of 11 concussed collegiate ice hockey players, the total SCAT2 score was no different from baseline following concussion. ${ }^{8}$ Similarly, in another small study of 10 concussed athletes, the postinjury SCAT3 memory score did not differ significantly from baseline. ${ }^{53}$

\section{Predicting Outcomes with SCAT Sideline Assessment}

Several authors have ventured to correlate sideline test scores and eventual outcomes following concussion. $\mathrm{Pu}-$ tukian et al. ${ }^{41}$ found that in collegiate athletes, a lower total SCAT2 score at the time of injury was correlated with more days to becoming symptom free $(\mathrm{p}<0.05)$ and more days until returning to graduated activity $(\mathrm{p}<0.05)$. In addition, a greater number of total symptoms was correlated with more days until becoming symptom free $(\mathrm{p}<$ $0.01)$ and returning to restricted activity $(\mathrm{p}<0.01)$ as well as returning to play $(\mathrm{p}<0.05)$. Notably, neither the SAC nor mBESS was significantly predictive of any of these outcomes ${ }^{44}$ which is similar to the findings of McCrea and coauthors. ${ }^{30}$ The group aimed to identify predictors of athletes who recover at greater than 7 days and found that in 570 athletes with concussion, 57 (10\%) of which had recovery outside of 1 week, the SAC or BESS did not predict a longer recovery, although a graded symptom scale did.

Baker et al. ${ }^{2}$ sought to elucidate predictors of difficulty returning to school in 91 student athletes aged 13-19 years old. Student athletes who struggled upon returning to school had significantly worse SCAT2 total scores than athletes who had no difficulty $(\mathrm{p}<0.003)$. Athletes who returned to school with difficulty also had significantly more symptoms with significantly worse severity than those who returned to school smoothly. There is no reported literature to date on the ability of the SCAT to specifically predict progression through the graduated return-to-play or learn protocols.

\section{Additional Baseline Testing Considerations}

Weber et al. ${ }^{55}$ studied the effects of dehydration on SCAT2 performance in 32 collegiate wrestlers. Their total SCAT2 score declined significantly from baseline to postpractice $(93.06$ vs $87.94 ; \mathrm{p}<0.05)$ and notably the number of errors recorded on the mBESS increased from 15.72 to $18.81(\mathrm{p}<0.05)$. Because the athletes' urine specific gravity increased from baseline to postpractice, the authors concluded that euhydration should be a goal prior to performance of sideline concussion tests.

Putukian et al. ${ }^{41}$ assessed whether anxiety or depression was associated with differences on the SCAT2. Both depressed and anxious patients were found to display worse total SCAT2 scores $(p<0.001)$. However, these differences appeared to be attributable to significantly more symptom reporting by these groups related to their psychiatric diagnoses, given the authors did not find that either diagnosis was related to cognitive (SAC) or balance (mBESS) components.

\section{Discussion}

We performed a systematic review of the literature pertaining to the SCAT and its major components (SAC and mBESS). After application of inclusion and exclusion criteria, 36 articles were reviewed in detail. Given the majority of data reported baseline or normative data, we focused on differences associated with age, sex, and concussion history, in addition to the ability to detect a concussion.

\section{Effect of Age}

Across the studies that analyzed baseline assessment results by age, the literature appears somewhat inconclusive. However, close examination finds that the studies reporting age differences on both the SCAT2 $2^{47}$ and $\mathrm{SAC}^{11}$ alone used large age ranges (e.g., 5-23 or 9-18 years old). In studies with more focused age ranges such as a pure high school (13-18 years old) ${ }^{18}$ or college ${ }^{57}$ population (18-23 years old), or comparing the two, ${ }^{33}$ there are few reported differences. This discrepancy suggests that age differences are mainly related to differences between the youngest athletes (age < 12) and oldest athletes (age 15-18 or 18-23), which was confirmed for the SCAT2 by Snyder et al. utilizing post hoc analysis. ${ }^{47}$ Given that the ChildSCAT3 is now available for children age $<13$ years old, we recommend ensuring the use of this modified version rather than the adolescent/adult SCAT3.

That said, one of the large studies assessing the SCAT2 normative data in high school athletes found significant age differences. ${ }^{50}$ The difference was between 9 th graders and each of 10th, 11th, and 12th graders on both the total SCAT2 and mBESS; however, differences between the oldest 3 grades did not exist. This may suggest an exposure or practice effect, in that 9th graders are likely being tested with the SCAT2 for the first time, while student athletes in older grades have probably been exposed to the test previously. The study in question did not clarify any possible effect of previous testing. In an effort to obtain accurate age information, we caution future normative studies to recognize this limitation and clarify the testing status of young athletes enrolled in the study, as well as whether alternate forms of the SAC are being used across time intervals.

\section{Effect of Sex}

The association of sex and sideline assessment scoring appeared to depend on whether the components are used alone or if one examines the total SCAT2 composite score. While the evidence appears convincing that males perform slightly worse than females on the SAC ${ }^{19,50,51,57}$ and mBESS, $13,19,50$ it is important to note that these differences do not reliably translate to sex differences on the aggregate SCAT2. ${ }^{13,47,57}$ However, drawing firm conclusions on any sideline assessment score association with sex is difficult given the gross overrepresentation of male athletes across 
all studies (89.4\% SAC, 83.1\% SCAT2 and SCAT3). Future studies should continue to report sideline assessment normative data stratified by sex and ideally by sport, and preinjury baseline testing of athletes is preferable.

\section{Concussion History}

The current literature was inconclusive regarding whether prior concussion history is associated with differences on baseline sideline concussion assessments. The results ranged from athletes with previous concussions performing worse on the SCAT2 overall,,$^{50}$ to no difference, ${ }^{16,57}$ to paradoxically performing better on all cognitive testing. ${ }^{45,46}$ The observation that a larger percentage of previously concussed than never concussed athletes were able to successfully perform the more difficult components of the original SCAT suggests the possibility of an exposure effect, in that athletes who suffered previous concussions were exposed to the SCAT. ${ }^{45,46}$ Also, given the exposure effect, it is possible that a test ceiling effect also exists, as is the case with brief cognitive screening examinations. Further research is needed to understand the significance of prior concussion on sideline assessment, as well as to clarify whether separate normative data are needed.

\section{Detecting Concussion}

Based on this review, there are important limitations inherent in applying the literature utilizing the SAC to a given concussed athlete. A 1-point decline on the SAC has been reported to be $95 \%$ sensitive and $76 \%$ specific for detecting concussion. ${ }^{3,28}$ However, it is notable that these articles used a population of male high school and college football players exclusively., ${ }^{3,28}$ While the specific study population is a strength in applying results to male football players, the generalizability to other sports may be limited. This has been demonstrated as the SAC has been shown to be less efficacious in detecting concussion in a mixed sex and sports sample (mixed sports, $\mathrm{AUC}=0.68$, vs football, AUC > 0.9) ${ }^{3,11}$ Given that only a single prospective investigation of the sensitivity and specificity of the SCAT2 ${ }^{41}$ (in rugby only) is available and none is available for the SCAT3, this is an area in need of further prospective studies of large, mixed sport and sex cohorts. Finally, it should be emphasized that the reliability and ultimate utility of the SCAT (and its quantitative component parts) are based on valid test results, which require good faith effort on the part of the athlete taking the test at any time point. If an athlete were to give a minimal effort at baseline and a full effort following concussion, the result may be an undetected concussion. While some studies have addressed "sandbagging," or purposeful suboptimal performance on computerized baseline neurocognitive testing, ${ }^{48}$ no study to date has addressed this matter with the SCAT.

\section{Review Limitations}

One goal of the present review was to aggregate normative data in an attempt to have age- and sex-specific norms compiled from a sport-, geographic-, and setting-diverse population. However, we found this to be difficult due to a wide variation in reporting of age ranges (e.g., 13-15 vs
14-16 vs $13-18$ ), which could not be reliably aggregated. Additionally, the literature reviewed was rarely stratified by age and sex simultaneously, which effectively means utilizing the same norms for both sexes in a given age group. This is problematic given the findings presented above regarding sex differences. Finally, we focused this review solely on the athlete population, which means important literature may not have been assessed due to the exclusion of nonathletes, such as military personnel.

\section{Conclusions and Future Directions}

The SCAT and all its iterations have provided the groundwork for major improvements in the acute diagnosis of a concussion. Between all 3 iterations, athletes at all levels are being tested with the SCAT. Although we could not calculate aggregate normative data, one reason may be due to a significant strength of the SCAT, the constant evolution of the test to be more comprehensive and complete.

Looking toward the fifth Concussion in Sport Group meeting in Berlin in 2016, one may expect further revision to the SCAT3. However, based on this systematic review, we believe that further study of this concussion-screening tool is warranted. Based on our review, areas recommended for further investigation include: 1) the ability of the SCAT (as an instrument) and its individual components to detect acute concussion on the sideline (with and without baseline test availability); 2) the utility of the SCAT and its components as serial assessments to monitor recovery, including investigations aimed at assessing the SCAT as a tool for predicting progression through return-to-play protocols; 3 ) the effects of repeated exposure to the SCAT, including the use of alternate forms of its components, as well as possible ceiling effects on component and total scores; and 4) the effects of age, sex, sport, and concussion history on total SCAT and individual component scores.

\section{References}

1. Alla S, Sullivan SJ, Hale L, McCrory P: Self-report scales/ checklists for the measurement of concussion symptoms: a systematic review. Br J Sports Med 43 (Suppl 1):i3-i12, 2009

2. Baker JG, Leddy JJ, Darling SR, Rieger BP, Mashtare TL, Sharma T, et al: Factors associated with problems for adolescents returning to the classroom after sport-related concussion. Clin Pediatr (Phila) 54:961-968, 2015

3. Barr WB, McCrea M: Sensitivity and specificity of standardized neurocognitive testing immediately following sports concussion. J Int Neuropsychol Soc 7:693-702, 2001

4. Bonfield CM, Wecht DA, Lunsford LD: Concussion in ice hockey. Prog Neurol Surg 28:161-170, 2014

5. Curaudeau GA, Sharma N, Rovin RA: Development of an iPhone application for sideline concussion testing. Neurosurg Focus 31(5):E4, 2011

6. Daniel JC, Nassiri JD, Wilckens J, Land BC: The implementation and use of the SAC at the US Naval Academy. Mil Med 167:873-876, 2002

7. Echlin PS, Johnson AM, Holmes JD, Tichenoff A, Gray S, Gatavackas H, et al: The Sport Concussion Education Project. A brief report on an educational initiative: from concept to curriculum. J Neurosurg 121:1331-1336, 2014

8. Echlin PS, Johnson AM, Riverin S, Tator CH, Cantu RC, Cusimano MD, et al: A prospective study of concussion education in 2 junior ice hockey teams: implications for sports concussion education. Neurosurg Focus 29(5):E6, 2010 
9. Echlin PS, Skopelja EN, Worsley R, Dadachanji SB, LloydSmith DR, Taunton JA, et al: A prospective study of physician-observed concussion during a varsity university ice hockey season: incidence and neuropsychological changes. Part 2 of 4. Neurosurg Focus 33(6):E2, 1-11, 2012

10. Echlin PS, Tator CH, Cusimano MD, Cantu RC, Taunton JE, Upshur RE, et al: A prospective study of physician-observed concussions during junior ice hockey: implications for incidence rates. Neurosurg Focus 29(5):E4, 2010

11. Galetta KM, Morganroth J, Moehringer N, Mueller B, Hasanaj L, Webb N, et al: Adding vision to concussion testing: a prospective study of sideline testing in youth and collegiate athletes. J Neuroophthalmol 35:235-241, 2015

12. Galetta MS, Galetta KM, McCrossin J, Wilson JA, Moster S, Galetta SL, et al: Saccades and memory: baseline associations of the King-Devick and SCAT2 SAC tests in professional ice hockey players. J Neurol Sci 328:28-31, 2013

13. Glaviano NRBS, Benson S, Goodkin HP, Broshek DK, Saliba S: Baseline SCAT2 assessment of healthy youth studentathletes: preliminary evidence for the use of the ChildSCAT3 in children younger than 13 years. Clin J Sport Med 25:373-379, 2015

14. Guskiewicz KM, Register-Mihalik J, McCrory P, McCrea M, Johnston K, Makdissi M, et al: Evidence-based approach to revising the SCAT2: introducing the SCAT3. Br J Sports Med 47:289-293, 2013

15. Gysland SM, Mihalik JP, Register-Mihalik JK, Trulock SC, Shields EW, Guskiewicz KM: The relationship between subconcussive impacts and concussion history on clinical measures of neurologic function in collegiate football players. Ann Biomed Eng 40:14-22, 2012

16. Hanninen T, Tuominen M, Parkkari J, Vartiainen M, Ohman $\mathrm{J}$, Iverson GL, et al: Sport concussion assessment tool-3rd edition-normative reference values for professional ice hockey players. J Sci Med Sport [epub ahead of print], 2015

17. Harmon KG, Drezner JA, Gammons M, Guskiewicz KM, Halstead M, Herring SA, et al: American Medical Society for Sports Medicine position statement: concussion in sport. Br J Sports Med 47:15-26, 2013

18. Hunt TN, Ferrara MS: Age-related differences in neuropsychological testing among high school athletes. J Athl Train 44:405-409, 2009

19. Jinguji TM, Bompadre V, Harmon KG, Satchell EK, Gilbert K, Wild J, et al: Sport Concussion Assessment Tool-2: baseline values for high school athletes. Br J Sports Med 46:365-370, 2012

20. Kerr ZY, Register-Mihalik JK, Marshall SW, Evenson KR, Mihalik JP, Guskiewicz KM: Disclosure and non-disclosure of concussion and concussion symptoms in athletes: review and application of the socio-ecological framework. Brain Inj 28:1009-1021, 2014

21. King D, Brughelli M, Hume P, Gissane C: Assessment, management and knowledge of sport-related concussion: systematic review. Sports Med 44:449-471, 2014

22. King D, Brughelli M, Hume P, Gissane C: Concussions in amateur rugby union identified with the use of a rapid visual screening tool. J Neurol Sci 326:59-63, 2013

23. King D, Gissane C, Hume PA, Flaws M: The King-Devick test was useful in management of concussion in amateur rugby union and rugby league in New Zealand. J Neurol Sci 351:58-64, 2015

24. King LA, Horak FB, Mancini M, Pierce D, Priest KC, Chesnutt $\mathrm{J}$, et al: Instrumenting the balance error scoring system for use with patients reporting persistent balance problems after mild traumatic brain injury. Arch Phys Med Rehabil 95:353-359, 2014

25. Krol AL, Mrazik M, Naidu D, Brooks BL, Iverson GL: Assessment of symptoms in a concussion management programme: method influences outcome. Brain Inj 25:13001305,2011
26. Kurowski BG, Pomerantz WJ, Schaiper C, Ho M, Gittelman MA: Impact of preseason concussion education on knowledge, attitudes, and behaviors of high school athletes. J Trauma Acute Care Surg 79 (3 Suppl 1):S21-S28, 2015

27. Langlois JA, Rutland-Brown W, Wald MM: The epidemiology and impact of traumatic brain injury: a brief overview. J Head Trauma Rehabil 21:375-378, 2006

28. McCrea M: Standardized mental status testing on the sideline after sport-related concussion. J Athl Train 36:274-279, 2001

29. McCrea M, Guskiewicz KM, Marshall SW, Barr W, Randolph C, Cantu RC, et al: Acute effects and recovery time following concussion in collegiate football players: the NCAA Concussion Study. JAMA 290:2556-2563, 2003

30. McCrea M, Guskiewicz K, Randolph C, Barr WB, Hammeke TA, Marshall SW, et al: Incidence, clinical course, and predictors of prolonged recovery time following sport-related concussion in high school and college athletes. J Int Neuropsychol Soc 19:22-33, 2013

31. McCrea M, Kelly JP, Kluge J, Ackley B, Randolph C: Standardized assessment of concussion in football players. Neurology 48:586-588, 1997

32. McCrea M, Kelly JP, Randolph C, Cisler R, Berger L: Immediate neurocognitive effects of concussion. Neurosurgery 50:1032-1042, 2002

33. McCrea M, Kelly JP, Randolph C, Kluge J, Bartolic E, Finn $\mathrm{G}$, et al: Standardized assessment of concussion (SAC): onsite mental status evaluation of the athlete. J Head Trauma Rehabil 13:27-35, 1998

34. McCrory P, Johnston K, Meeuwisse W, Aubry M, Cantu R, Dvorak J, et al: Summary and agreement statement of the 2nd International Conference on Concussion in Sport, Prague 2004. Br J Sports Med 39:196-204, 2005

35. McCrory P, Meeuwisse W, Johnston K, Dvorak J, Aubry M, Molloy M, et al: Consensus Statement on Concussion in Sport: the 3rd International Conference on Concussion in Sport held in Zurich, November 2008. Br J Sports Med 43 (Suppl 1):i76-i90, 2009

36. McCrory P, Meeuwisse WH, Aubry M, Cantu B, Dvorák J, Echemendia RJ, et al: Consensus statement on concussion in sport: the 4th International Conference on Concussion in Sport held in Zurich, November 2012. Br J Sports Med 47:250-258, 2013

37. Miller JR, Adamson GJ, Pink MM, Sweet JC: Comparison of preseason, midseason, and postseason neurocognitive scores in uninjured collegiate football players. Am J Sports Med 35:1284-1288, 2007

38. Moher D, Liberati A, Tetzlaff J, Altman DG: Preferred reporting items for systematic reviews and meta-analyses: the PRISMA statement. J Clin Epidemiol 62:1006-1012, 2009

39. Okonkwo DO, Tempel ZJ, Maroon J: Sideline assessment tools for the evaluation of concussion in athletes: a review. Neurosurgery 75 (Suppl 4):S82-S95, 2014

40. Putukian M: The acute symptoms of sport-related concussion: diagnosis and on-field management. Clin Sports Med 30:49-61, viii, 2011

41. Putukian M, Echemendia R, Dettwiler-Danspeckgruber A, Duliba T, Bruce J, Furtado JL, et al: Prospective clinical assessment using Sideline Concussion Assessment Tool-2 testing in the evaluation of sport-related concussion in college athletes. Clin J Sport Med 25:36-42, 2015

42. Ruhe A, Gänsslen A, Klein W: The incidence of concussion in professional and collegiate ice hockey: are we making progress? A systematic review of the literature. Br J Sports Med 48:102-106, 2014

43. Sabin MJ, Van Boxtel BA, Nohren MW, Broglio SP: Presence of headache does not influence sideline neurostatus or balance in high school football athletes. Clin J Sport Med 21:411-415, 2011 
44. Schmitt DM, Hertel J, Evans TA, Olmsted LC, Putukian M: Effect of an acute bout of soccer heading on postural control and self-reported concussion symptoms. Int J Sports Med 25:326-331, 2004

45. Schneider KJ, Emery CA, Kang J, Schneider GM, Meeuwisse WH: Examining Sport Concussion Assessment Tool ratings for male and female youth hockey players with and without a history of concussion. Br J Sports Med 44:1112-1117, 2010

46. Shehata N, Wiley JP, Richea S, Benson BW, Duits L, Meeuwisse WH: Sport Concussion Assessment Tool: baseline values for varsity collision sport athletes. Br J Sports Med 43:730-734, 2009

47. Snyder AR, Bauer RM: A normative study of the Sport Concussion Assessment Tool (SCAT2) in children and adolescents. Clin Neuropsychol 28:1091-1103, 2014

48. Szabo AJ, Alosco ML, Fedor A, Gunstad J: Invalid performance and the ImPACT in National Collegiate Athletic Association Division I football players. J Athl Train 48:851855,2013

49. Valovich TC, Perrin DH, Gansneder BM: Repeat administration elicits a practice effect with the balance error scoring system but not with the standardized assessment of concussion in high school athletes. J Athl Train 38:51-56, 2003

50. Valovich McLeod TC, Bay RC, Lam KC, Chhabra A: Representative baseline values on the Sport Concussion Assessment Tool 2 (SCAT2) in adolescent athletes vary by gender, grade, and concussion history. Am J Sports Med 40:927933, 2012

51. Valovich McLeod TC, Barr WB, McCrea M, Guskiewicz KM: Psychometric and measurement properties of concussion assessment tools in youth sports. J Athl Train 41:399408, 2006

52. Valovich McLeod TC, Perrin DH, Guskiewicz KM, Shultz SJ, Diamond R, Gansneder BM: Serial administration of clinical concussion assessments and learning effects in healthy young athletes. Clin J Sport Med 14:287-295, 2004

53. Vernau BT, Grady MF, Goodman A, Wiebe DJ, Basta L, Park Y, et al: Oculomotor and neurocognitive assessment of youth ice hockey players: baseline associations and observations after concussion. Dev Neuropsychol 40:7-11, 2015

54. Wang Y, Nelson LD, LaRoche AA, Pfaller AY, Nencka AS, Koch KM, et al: Cerebral blood flow alterations in acute sport-related concussion. J Neurotrauma [epub ahead of print], 2015
55. Weber AF, Mihalik JP, Register-Mihalik JK, Mays S, Prentice WE, Guskiewicz KM: Dehydration and performance on clinical concussion measures in collegiate wrestlers. J Athl Train 48:153-160, 2013

56. Yengo-Kahn AM, Johnson DJ, Zuckerman SL, Solomon GS: Concussions in the National Football League: a current concepts review. Am J Sports Med [epub ahead of print], 2015

57. Zimmer A, Marcinak J, Hibyan S, Webbe F: Normative values of major SCAT2 and SCAT3 components for a college athlete population. Appl Neuropsychol Adult 22:132-140, 2015

58. Zimmer A, Piecora K, Schuster D, Webbe F: Sport and team differences on baseline measures of sport-related concussion. J Athl Train 48:659-667, 2013

\section{Disclosures}

Dr. Solomon receives consulting fees from the Tennessee Titans (NFL), the Nashville Predators (NHL), and the athletic departments for several universities. In addition he is a member of the ImPACT advisory board and receives reimbursement for expenses related to board meetings, and also receives royalties from book sales. Dr. Sills is a consulting physician to the Nashville Predators (uncompensated) and also serves as an unaffiliated neurotrauma consultant for the NFL.

\section{Author Contributions}

Conception and design: Yengo-Kahn, Zuckerman, Solomon. Acquisition of data: Yengo-Kahn, Hale, Zalneraitis, Zuckerman. Analysis and interpretation of data: Yengo-Kahn, Hale, Zalneraitis, Zuckerman, Solomon. Drafting the article: Yengo-Kahn, Hale, Zalneraitis. Critically revising the article: Yengo-Kahn, Zuckerman, Sills, Solomon. Reviewed submitted version of manuscript: all authors. Approved the final version of the manuscript on behalf of all authors: Yengo-Kahn. Administrative/technical/ material support: Solomon. Study supervision: Zuckerman, Sills, Solomon.

\section{Correspondence}

Aaron Yengo-Kahn, Vanderbilt Sports Concussion Center, Vanderbilt University School of Medicine, Village at Vanderbilt, 1500 21st Ave. S, Ste. 1506, Nashville, TN 37232. email: aaron.m.yengo-kahn@vanderbilt.edu. 